\title{
Improved image inpainting exemplar-based algorithms by boundary priori-knowledge
}

\author{
Junhong Zhao*, Jintao Tan, Yaobin Huang, and Chuanlong Lu \\ School of Automation Science and Engineering South China University of Technology, Guangzhou, \\ China
}

\begin{abstract}
Image inpainting plays an important role in restoration of cultural relics, pictures beautification. Criminisi algorithm creates good results in large-area inpainting. However, it does still have some deficiencies such as over-extending. In this paper, two improved algorithms based on prior knowledge of the boundary had been proposed by simulating the idea of manual repairing. An algorithm, by simulating the strategy that the next inpainted pixel will be near to the prior one, named nearer neighbor first algorithm, can void the random bounding of the to-be-inpainted pixle. Another algorithm, by simulating the strategy that the inpainting process, named no-inpainted first algorithm, will be in multiple directions, can void the inpainting process in a single direction. The results reveal that the neighborhood-first algorithm performs better than Criminsi algorithm in repairing the missing structure while the unrepaired-first algorithm performs better than Criminsi algorithm in repairing the missing texture.
\end{abstract}

Keywords: Image inpainting, Exemplar-Based, Fill-front, PrioriKnowledge.

\section{Introduction}

Image inpainting is widely used in repairing damaged works of art. With the widespread application of digital images, it is necessary to research image repaired of digital images, which enables it to meet the needs of photo beautification and removal of specific objects in film and television special effects production. The existing algorithms include the deep learning-based methods and the traditional image methods. The traditional algorithms can be classified into two categories:(1) small region inpainting methods based on partial differential equations such as BSCB model and CDD model and(2) large region inpainting methods based on exemplar such as Criminisi algorithm, which use the structure and data information to design a priority function to select a best inpainting pixel ${ }^{[1]}$. The deep learning-based methods include auto encoders methods and convolutional neural networks methods ${ }^{[2-4]}$. The deep-learning methods require high computing overhead, while the traditional algorithm is the image inpainting algorithm based on sample block, which is widely used due to its ability to remove and repair large areas of objects, simple implementation and reasonable results. However, there are some shortcomings. Improvement had made which would be

* Corresponding author: jhzhao@scut.edu.cn 
divided into three categories. The first category is an improvement to the priority model of Criminisi algorithm to obtain a better filling order, which can reduce structure propagation errors and accumulated errors ${ }^{[2-14]}$. The second category improves the patch matching method to reduce the uncoordinated block boundary ${ }^{[2,5,6,7,10,12]}$. The last category improves the speed by improving the search strategy ${ }^{[3,5,8,9,11,13]}$.

Please follow these instructions as carefully as possible so all articles within a conference have the same style to the title page. This paragraph follows a section title so it should not be indented.

\section{Exemplar-based image inpainting algorithm}

Criminisi's image repaired algorithm based on sample patches ${ }^{[14]}$ is shown in Figure 1.I is the image to be repaired, which includes the target region $\Omega$, the source region $\Phi(\Phi=I-$ $\Omega$ )and the contour $\delta \Omega$.

The steps of Criminisi algorithm are as follow. First, the priority of all points, $\boldsymbol{p}$, on $\boldsymbol{\delta} \boldsymbol{\Omega}$ will be calculated, and the block containing the point $\boldsymbol{p}$ with the largest priority is selected as the block $\psi \boldsymbol{p}$ to be inpainting. Next, in the source region $\Phi$, the best matching block $\boldsymbol{\psi} \boldsymbol{q}$ that has the smallest sum of error squares of all pixels (SSD, Sum of Squared Differential) with the block $\boldsymbol{\psi} \boldsymbol{p}$ to be inpainting is selected. Then, the corresponding pixel of block $\boldsymbol{\psi} \boldsymbol{q}$ is filled into the block $\psi \boldsymbol{p}$ to be inpainting, and the filling front $\boldsymbol{\delta} \boldsymbol{\Omega}$ is updated. The cycle about the above steps is repeated until the region $\boldsymbol{\Omega}$ is empty.
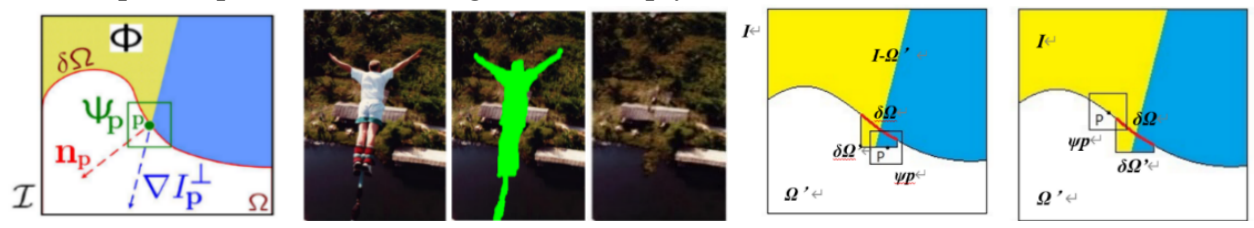

Fig. 1.Criminisi Fig. 2. The inpainting effect

Fig. 3. Neighborhood-first Fig. 4. Unrepaired-first

The priority formula of Criminisi's algorithm is defined as follows:

$$
P(p)=C(p) \cdot D(p)
$$

In Equation (1), $\boldsymbol{C}(\boldsymbol{p})$ is the confidence term, $\boldsymbol{D}(\boldsymbol{p})$ is the data term. The definitions of them are as follow:

$$
C(p)=\frac{\sum_{q \in \boldsymbol{\Psi} p \cap(I-\Omega)} C(q)}{|\Psi p|} \quad D(p)=\frac{\left|\nabla I_{p}^{\frac{1}{p}} \cdot n_{p}\right|}{\alpha}
$$

In Equation (2), where $|\boldsymbol{\psi} \boldsymbol{p}|$ is the area of $\boldsymbol{\psi} \boldsymbol{p}, \boldsymbol{n}_{\boldsymbol{p}}$ is a unit vector orthogonal to the front $\boldsymbol{\delta} \boldsymbol{\Omega}$ in the point $\boldsymbol{p}$ and $\boldsymbol{\alpha}$ is a normalization factor, which usually be taken to 255 . For $\boldsymbol{C}(\boldsymbol{p})$ and $\nabla I_{P}$ we have:

$$
C(q)=\left\{\begin{array}{cc}
1 & q \notin \Omega \\
0 & q \in \Omega
\end{array} \quad \nabla I_{P}=\frac{\left(-I_{y}, I_{x}\right)}{\sqrt{I_{x}^{2}+I_{y}^{2}}}\right.
$$

$\perp$ represents a vertical operation. $I_{x}$ and $I_{y}$ represents the deviation of point $\boldsymbol{p}$ in the $\boldsymbol{x}$ and $\boldsymbol{y}$ directions. $\nabla I_{p}{ }^{\perp}$ represents the direction of the equipotential line of point $\boldsymbol{p}$ in the source region. After the priority that calculating of the traversal of point $\boldsymbol{p}$ on the boundary $\boldsymbol{\delta} \boldsymbol{\Omega}$ using Equation (1), the point $\boldsymbol{p}$ with the highest priority is selected. Then the block $\Psi_{\hat{p}}$ to be inpainted is formed with this point $\boldsymbol{p}$ as the center, and the matching block $\Psi_{\hat{q}}$ that is most similar to it is found in the source region $\boldsymbol{\Phi}$. The equation to measure the similarity between $\Psi_{\hat{p}}$ and $\Psi_{q}$ is:

$$
\Psi_{\hat{q}}=\underset{\Psi_{q} \in \Phi}{\operatorname{argmin}} d\left(\Psi_{\hat{p}}, \Psi_{q}\right)
$$


In Equation (4), $d\left(\Psi_{\hat{p}}, \Psi_{q}\right)$ is defined as the sum of error squares (SSD) of the filled pixels in two region blocks. $d\left(\Psi_{\hat{p}}, \Psi_{q}\right)$ is the SSD of the pixels of the filled part of the block $\Psi_{\hat{p}}$ to be inpainted and the corresponding part of $\Psi_{q}$. After finding the most similar matching block $\Psi_{\hat{q}}$, fill the pixels of the corresponding region in $\Psi_{\hat{q}}$ into $\Psi_{\hat{p}} \cap \boldsymbol{\Omega}$, and update the confidence term to get the new $\boldsymbol{C}(\boldsymbol{p})$, then repeat the above step until the filling is completed.

This algorithm can achieve the effect of automatic object removal and inpainting. However, there are also some shortcomings, such as an unreasonable stretch of green space towards the sea and an unreasonable rock pile filling above the roof of the house in fig2.Therefore, there are many ways to improve this algorithm. One of the important ideas is to turn the product of $\boldsymbol{C}(\boldsymbol{p})$ and $\boldsymbol{D}(\boldsymbol{p})$ in the priority formula into a summation which can prevent the difference of $\boldsymbol{D}(\boldsymbol{p})$ from being reduced due to the rapid reduction of $\boldsymbol{C}(\boldsymbol{p})$ to 0 . This improvement can reduce the randomness of the selection of repair points.

Therefore, many improvements are proposed. One of the important ideas is to change the product of $\boldsymbol{C}(\boldsymbol{p})$ and $\boldsymbol{D}(\boldsymbol{p})$ in the priority formula into a summation. Through this method, the problems of zero product and small difference of $\boldsymbol{D}(\boldsymbol{p})$ due to the rapid decrease of $\boldsymbol{C}(\boldsymbol{p})$ to zero are solved, and the randomness of the selection of repair points is reduced.Based on the idea of summing $\boldsymbol{C}(\boldsymbol{p})$ and $\boldsymbol{D}(\boldsymbol{p})$, the paper simulates the ideas of artificial repair, which are to use the previous filling front as prior knowledge to improve the algorithm. The specific implementations are as follows.

\section{The improved method of neighborhood-first}

Usually, painters do picture inpainting along with the last inpainted boundary from outside to inside. The two sequence operations will probably be in the same small area and the latest to-be-inpainted pixel will be near to the last one. The algorithm will get the difference between the two boundaries to modify the priority item $P(p)$. An improved method of 'neighborhood-first' will be described as follow.

InFig3, $\boldsymbol{I}$ is the whole image. The white part is the area to be filled $\left(\boldsymbol{\Omega}^{\prime}\right)$. The color part is the existing image part $\left(\boldsymbol{I}-\boldsymbol{\Omega}^{\prime}\right)$. The square block centered on point $\boldsymbol{p}$ is the patch to be filled $(\psi p) . \delta \Omega$ is the filling front of the previous restoration.

The red line indicates the inpainted part of $\boldsymbol{\delta} \boldsymbol{\Omega}$. The intersection line is the black line of $\boldsymbol{\delta} \boldsymbol{\Omega}$. The black line indicates the current filling front $\boldsymbol{\delta} \boldsymbol{\Omega}$ '.

Define the new prior knowledge term $\boldsymbol{E}(\boldsymbol{p})$ as follows:

$$
E(p)=\frac{\sum_{q \in \Psi_{p} \cap\left(I-\Omega^{\prime}\right)} E(q)}{\left|\Psi_{P} \cap\left(I-\Omega^{\prime}\right)\right|} \quad E(q)= \begin{cases}0 & q \notin \delta \Omega \\ 1 & q \in \delta \Omega\end{cases}
$$

where $\mathrm{q}$ is the intersection of to-be filled are $\psi \boldsymbol{p}$ and the existing iamege part

A new modified priority calculation formula was as

$$
\begin{gathered}
P(p)=m C(p)+n D(p)+k E(p) \\
\boldsymbol{m}+\boldsymbol{n}+\boldsymbol{k}=1,0<\boldsymbol{m}<1,0<\boldsymbol{n}<1,0<\boldsymbol{k}<1
\end{gathered}
$$

where $\boldsymbol{E}(\boldsymbol{p})$ will be zero in the beginning inpainting step.

Form the definition above, $\boldsymbol{E}(\boldsymbol{p})$ will be bigger when the number of pixels which is belonging to previous boundary is bigger. In other words, the to-be-inpainted boundary is near to the previous boundary. So, the improved algorithm is named Neighborhood-first method. It can make the inpainting be in well-ordered to release the randomness.

\section{The improved method of " unrepaired-first "}

In fig4, $\boldsymbol{I}, \boldsymbol{\Omega}, \boldsymbol{\psi p} \boldsymbol{p}, \boldsymbol{p}$, and $\boldsymbol{\delta} \boldsymbol{\Omega}$ are the same definitions as the above. The color part is the existing image part. The black line indicates the current filling front $\delta \boldsymbol{\Omega}^{\prime} . \boldsymbol{\delta} \boldsymbol{\Omega}$ and $\boldsymbol{\delta} \boldsymbol{\Omega}$ ' have 
intersecting parts in the figure, which is the non-red line of $\boldsymbol{\delta} \boldsymbol{\Omega}$. Define the new prior knowledge terms $\boldsymbol{B}(\boldsymbol{p})$ and $\boldsymbol{H}(\boldsymbol{p})$ as follows:

$$
H(p)=C(p)+B(p)
$$

where $\boldsymbol{C}(\boldsymbol{p})$ is the confidence term of the original Criminisi algorithm, which the calculation method remains unchanged. For $\boldsymbol{B}(\boldsymbol{p})$, there is :

$$
B(p)= \begin{cases}b & p \in \delta \Omega \cap \delta \Omega^{\prime} \\ 0 & p \notin \delta \Omega \cap \delta \Omega^{\prime}\end{cases}
$$

There $\boldsymbol{b}$ is a specific parameter $(0<\boldsymbol{b}<1)$, which represents the weight value brought by point $\boldsymbol{p}$ on the previous filling front $\boldsymbol{\delta} \boldsymbol{\Omega}$. In the first inpainting step, there is no filling front of the previous one, so it can be considered that $\boldsymbol{\delta} \boldsymbol{\Omega}=\boldsymbol{\delta} \boldsymbol{\Omega}$ ', then $\boldsymbol{B}(\boldsymbol{p})=\boldsymbol{b}$.

In fig4, point $\mathbf{p}$ is on both the current filling front $\boldsymbol{\delta} \boldsymbol{\Omega}^{\prime}$ and the previous filling front $\boldsymbol{\delta} \boldsymbol{\Omega}$, so the $\boldsymbol{B}(\boldsymbol{p})$ of the $\boldsymbol{p}$ point is taken as $\boldsymbol{b}$. The new priority calculation formula is defined as:

$$
P(p)=\alpha H(p)+\beta D(p) \quad 2 \alpha+\beta=1, \alpha>0, \beta>0
$$

Whether each pixel on the current filling front $\delta \boldsymbol{\Omega}^{\prime}$ is still on the previous filling front $\boldsymbol{\delta} \boldsymbol{\Omega}$ is judged through the algorithm. If it is, a certain weight value would be given in the priority calculation. In this way, compared with the pixels that did not fill the front $\boldsymbol{\delta} \boldsymbol{\Omega}$ in the previous time, there is a $\boldsymbol{B}(\boldsymbol{p})$ term to increase the priority value, which is conducive to repair the pixels firstly that exist at both $\delta \Omega^{\prime}$ and $\delta \Omega$ (point $\mathrm{p}$ in Figure 4). Judging whether it has been filled, the possibility of filling in only one direction will be reduced.

\section{Results and comparisons}

The process of getting the picture to be repaired is to manually fill the area to be filled with the color $(0,255,0)$ on the source picture, which is pure green in the RGB color. $\boldsymbol{b}=0.5, \boldsymbol{\alpha}=0.3$, $\boldsymbol{\beta}=0.4$.

\subsection{Experimental images inpainting focusing on textures}

The following experiments will be on the pictures with strong texture structures.

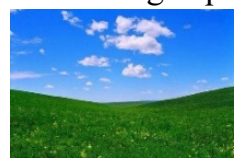

(a)

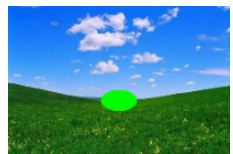

(b)

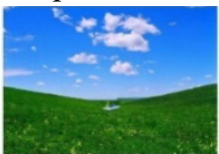

(c)

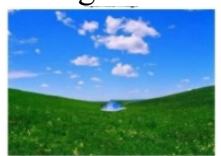

(d)

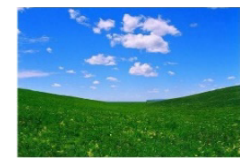

(e)

Fig. 5. the comparison of Criminisi's algorithm and two improved algorithms of picture5. (a) picture5 (b) to-be inpainted (c)Criminisi (d) Neighborhood-first (e) unrepaired-first

The area to be inpainted in fig5 spans two textures in vertical direction. (i.e., the sky and the grassland.) This inpainting would be achieved by extending the texture in the horizontal direction. By observing the inpainting results in fig5, it can be found that Criminisi algorithm and Neighborhood-first algorithm in fig5 (c) (d) extended from the vertical direction, and unreasonable extension of sky texture appears in the grassland region, and structures near sky clouds are used to repair the structure of grassland region. In fig5 (e), the boundary between the sky and the grassland is relatively clear, and the horizontal texture of the grassland is effectively extended, with no obvious difference from the original image boundary. Thus, the image texture can be effectively and reasonably filled to meet the expectation of inpainting. 


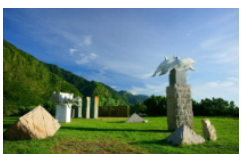

(a)

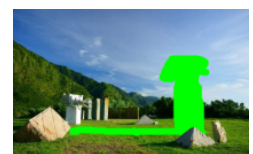

(b)

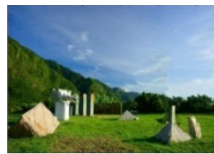

(c)

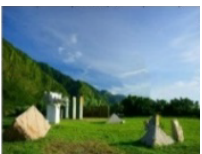

(d)

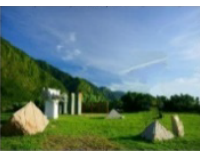

(e)

Fig. 6. The comparison of Criminisi's algorithm and two improved algorithms of picture6. (a) picture6 (b) to-be inpainted (c)Criminisi (d) Neighborhood-first (e) unrepaired-first

Observing the various inpainting results in Figure 6, an unreasonable continuation of the stone structure above the triangle in the lower right corner of Figure 6(c)(d) can be seen. There is no such continuation above the triangular stone in Figure 6(e), so the texture of the lawn is extended; although the boundary between dark grass and light grass is blurred, Figure 6 (c) (d) has neither the boundary between grass and lawn, but also the unreasonable extension of the structure. In contrast, the repair effect of Figure 6 (e) is better.

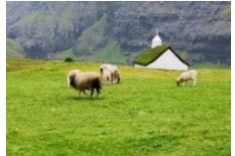

(a)

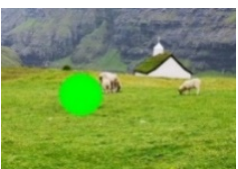

(b)

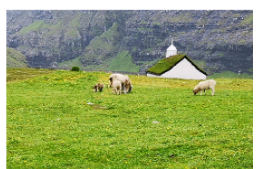

(c)

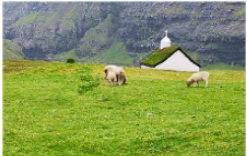

(d)

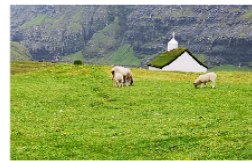

(e)

Fig. 7. The comparison of Criminisi's algorithm and two improved algorithms of picture 7 (a) picture7 (b) to-be inpainted (c)Criminisi (d) Neighborhood-first (e) unrepaired-first

The texture structure inconsistent with a part of the goat appears on the grass in the image after using the Criminisi's algorithm to repair, making the inpainted grass appear unreal and abrupt in fig7(c). The picture in fig7(e) looks more reasonable than in fig7(d) and more blurred. The area to be inpainted in Fig. 8 is on a light-colored lawn with only one texture, so the repair of the image can be obtained by extending the texture in all directions.

It can be seen that the texture of Fig. 8(c) did not extend as expected, so that one part of the sky in the upper part of the picture appeared in the lawn, and there was an obvious unreasonable structure. In Figure 8(d), one part of the shadow appeared in the lawn. In Figrue8 (e), the texture of the surrounding lawn is extended to the area to be repaired well and is almost indistinguishable from the original picture.

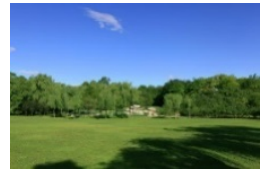

(a)

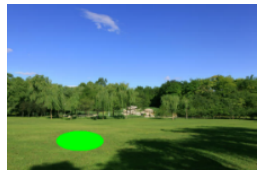

(b)

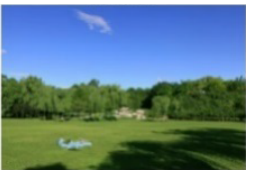

(c)

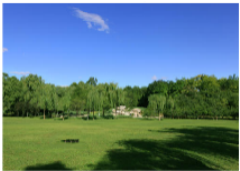

(d)

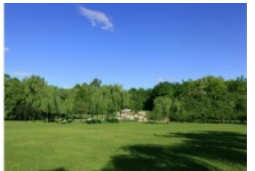

(e)

Fig. 8. The comparison of Criminisi's algorithm and two improved algorithms of picture 8 (a) picture8 (b) to-be inpainted (c)Criminisi (d) Neighborhood-first (e) unrepaired-first

Combined with the above inpainting effects and algorithm principles, the improved unrepaired-first algorithm can be selected for images with strong texture features, small proportion of structural features and no impact on the global situation of the source image. The improved unrepaired-first algorithm should be used for the image inpainting with low requirements for the structural features of the restored images.

\subsection{Experimental images inpainting focusing on structures}

The following experiment will be on the pictures with strong overlay structures. 


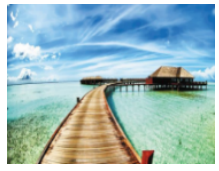

(a)

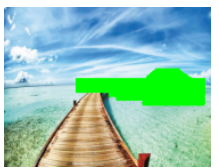

(b)

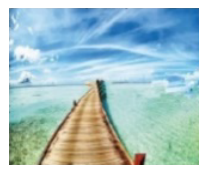

(c)

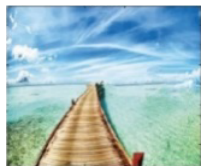

(d)

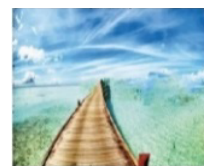

(e)

Fig. 9. The comparison of Criminisi's algorithm and two improved algorithms of picture 9 . (a) picture9 (b) to-be inpainted (c)Criminisi (d) Neighborhood-first (e) unrepaired-firs

Fig 9 shows that seashore cottages was inpainted automatically. Fig 9(d) shows a better effect on boundary propagation between the sea and the sky in a visually plausible way. Fig9(c) and Fig9(e) show some chaos.

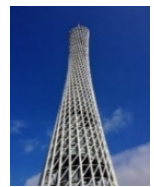

(a)

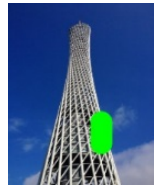

(b)

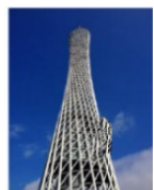

(c)

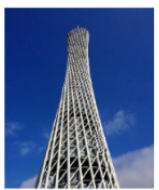

(d)

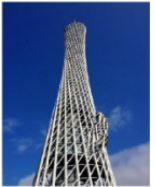

(e)

Fig. 10. The comparison of Criminisi's algorithm and two improved algorithms of picture10. (a) picture10 (b) to-be inpainted (c)Criminisi (d) Neighborhood-first (e) unrepaired-firs

In fig $10 b$, the to-be -inpainted area include the texture of the sky, the texture of the tower meshes and the structure of the tower contour lines. Fig 10c and Fig10e inpainted the sky by tower meshes. Fig 10d reveals a better effect because the algorithm make the inpainting point be near the latest point, which can emphasize the lines.

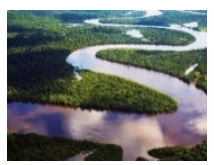

(a)

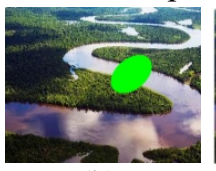

(b)

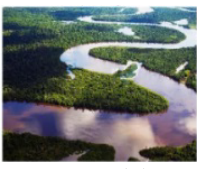

(c)

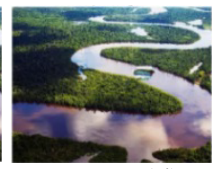

(d)

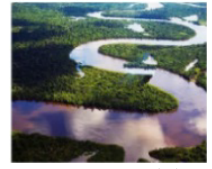

(e)

Fig. 11. The comparison of Criminisi's algorithm and two improved algorithms of picture11. (a) picture11 (b) to-be inpainted (c)Criminisi (d) Neighborhood-first (e) unrepaired-firs

In Fig $11 \mathrm{~b}$, the to-be -inpainted area include the shorelines, the textures of water surface and the textures of trees. an unreasonable propagation with tree textures and water plane textures from the left shoreline to the right shoreline can be found in fig $11 \mathrm{c}$ and fig $11 \mathrm{e}$. Fig $11 \mathrm{~d}$ reveals a better effect on inpainting the shorelines. Fig $12 \mathrm{~d}$ reveals a better effect on ring than fig12c and 12e.

Combined with the above inpainting effects and algorithm principles, the improved neighborhood-first algorithm can be selected for images with strong structure features. The improved neighborhood-first algorithm would be used for the image inpainting with low requirements for the textural features of the restored images.

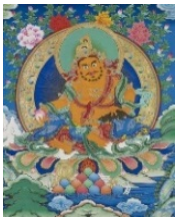

(a)

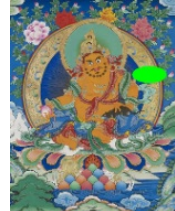

(b)

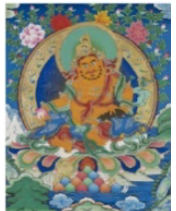

(c)

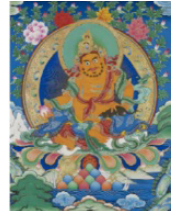

(d)

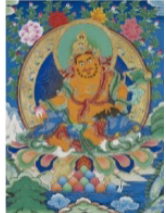

(e)

Fig. 12. The comparison of Criminisi's algorithm and two improved algorithms of picture12. (a) picture12 (b) to-be inpainted (c)Criminisi (d) Neighborhood-first (e) unrepaired-firs 


\section{Conclusion}

Combined with the above inpainting effects and algorithm principles, the improved algorithms can be selected for images inpainting by strong textural features or strong structural features.

The results reveal that the neighborhood-first algorithm performs better than Criminsi algorithm in repairing the missing structure while the unrepaired-first algorithm performs better than Criminsi algorithm in repairing the missing texture.

\section{References}

1. Zhao Lulu, Shen Ling, Hong Richang. Survey on Image Inpainting Research Progress[J]. Computer Science,2021,48(03):14-26

2. Wu Xiaojun, Li Gongqing. Large Scale Image Inpainting Based on Exemplar and Structure Information[J]. Acta Electronica Sinica, 2012, 40(8):1509-1514.

3. Zhang song, Zhou Xuya. An Improved Inpainting Algorithm with Variable-SizeExemplar [J]. Chinese Journal of Sensors and Actuators, 2012, 25(3):383-386.

4. Guo Yong, Wang Mei. Research on Exemplar-Based Digital Improving Image Inpainting Algorithms [J]. Software Guide, 2013, 12(10):156-158.

5. Xi X Y, Wang F L, Liu Y F. Improved Criminisi Algorithm Based on a New Priority Function with the Gray Entropy[C]// International Conference on Computational Intelligence and Security. IEEE, 2014:214-218.

6. $\mathrm{Hu} \mathrm{G}$, Xiong L. Criminisi-Based Sparse Representation for Image Inpainting[C]// IEEE Third International Conference on Multimedia Big Data. IEEE, 2017:389-393.

7. Zheng Yuting, Wu Jin. The Criminisi Algorithm Based on Ant Colony Optimization for Image Inpainting [J]. Infrared Technology, 2017, 39(3):221-225.

8. Zeng Jiexian, Wang Can. Image completion based on redefined priority and image division[J]. Journal of Image and Graphics, 2017, 22(9):1183-1193.

9. Chi Yue, He Ning, Zhang Qi. An Improved Method of Object Removal Based on Criminisi Method[J]. Journal of Beijing Union University, 2017, 31(1):67-74.

10. Du K, Domas S, Wu M, et al. A Hole-Filling Framework Based on DIBR and Improved Criminisi's Inpainting Algorithm for 3D Videos[C]. International Conference on Cloud and Big Data Computing. 2017:119-124.

11. Zhu Xiaolin, Chen Xiaodong, Zhu Yuanzhu,el. An Image Restoration Algorithm Based on Structure and Texture Synthesis with Reconstruction of Significant Structure of Images[J]. Journal of Graphics, 2014, 35(3):336-342.

12. Kwok T H, Sheung H, Wang C C L. Fast query for exemplar-based image completion[J]. IEEE Transactions on Image Processing A Publication of the IEEE Signal Processing Society, 2010, 19(12):3106-15.

13. Zhao Nan, Wang Huiqin,Wu Meng. Criminisi Digital Inpainting Algorithm Based on Markov Random Field Matching Criterion [J]. Journal of Frontiers of Computer Science and Technology, 2017, 11(7):1150-1158.

14. Criminisi A, Perez P, Toyama K. Region filling and object removal by exemplar-based image inpainting[J]. IEEE Transactions on Image Processing, 2004, 13(9):1200-1212. 